\title{
On a mechanism of intensification of field-aligned currents at the solar chromosphere-quiescent prominence boundaries
}

\author{
P. Nenovski ${ }^{1}$, V. N. Dermendjiev² ${ }^{2}$ M. Detchev ${ }^{2}$, J.-C. Vial ${ }^{3}$, and K. Bocchialini ${ }^{3}$ \\ 1 Institute of Geophysics Acad. G. Bonchev Str., Bl. 3, 1113 Sofia, Bulgaria \\ 2 Institute of Astronomy, 72 Tzarigradsko chaussee Blvd., 1784 Sofia, Bulgaria and Isaac Newton Institute of \\ Chile Bulgarian Branch (Prof. Dermendjiev deceased on January 2001) \\ 3 Institut d'Astrophysique Spatiale, Université Paris XI-CNRS, Bât. 121, 91405 Orsay Cedex, France \\ e-mail: vial@medoc-ias.u-psud.fr; bocchialini@medoc-ias.u-psud.fr
}

Received 1 September 2000 / Accepted 15 June 2001

\begin{abstract}
Field-aligned electric currents (FAC) are assumed to support various prominence configurations and it is usually supposed that the quiescent prominence (QP) destabilisation can be initiated by some plasma instability. In this work we study another possibility of QP destabilisation connected with FAC changes. Such a destabilisation may occur when FAC flowing along a loop-shaped QP structure are intensified by bouncing surface MHD modes, presumably generated by motions in the photosphere under, or adjacent to, the QP. The MHD disturbances at the prominence "feet" propagate upwards as Alfven waves and surface modes. We study the possibility that the generated MHD modes, which carry FAC, bounce in the loop-shaped QP structure, provided that they undergo multiple reflection at the prominence feet. This is an interesting physical situation leading to intensification of FAC and promising to be another source of QP destabilisation.
\end{abstract}

Key words. Sun: prominences - magnetohydrodynamics

\section{Introduction}

The subject of this study is the stability problem of field aligned currents (FAC) flowing along quiescent prominences (QPs). These prominences are located in the solar corona and are denser and cooler objects than their surroundings and relatively quiet if they are viewed at large scales. However, if we take into account their fine structure, they must be treated as dynamic and nonhomogenous formations. Also, they are imbedded in a hot corona via a so- called prominence-corona transition region. The precise knowledge of prominence plasma parameters (temperature, density, ionisation ratio and magnetic field) is very important for prominence theory and related problems (Tandberg-Hanssen 1995). Typical values of QP temperature lie in the range 5000-8000 K (Hirayama 1985a; Zhang et al. 1987; Mein \& Mein 1991). Lower temperatures $(T \sim 4300 \mathrm{~K})$ have been reported by Hirayama (1985b). An interesting tendency of increasing prominence temperature toward the outer edges, where temperatures may reach values of $10^{4}$ to $2 \times 10^{4} \mathrm{~K}$, has been pointed out by Hirayama (1971). The prominence plasma density is not as well known as the temperature because of

Send offprint requests to: P. Nenovski,

e-mail: nenovski@geophys.bas.bg observational difficulties. The electron density is in the range of $10^{10}-10^{11} \mathrm{~cm}^{-3}$ (Hirayama 1972), but these values have been found not to be precise (Vial 1986). The registered densities depend on both the prominence type and the method used (Hirayama 1985b). Electron densities ten times smaller (Bommier et al. 1986) and larger than $10^{11} \mathrm{~cm}^{-3}\left(N_{\mathrm{e}} \sim 10^{11.3} \mathrm{~cm}^{-3}\right)$ (Landman 1984) have been found. The hydrogen density of QP lies in the range of $3-6 \times 10^{11} \mathrm{~cm}^{-3}$ (Landman 1984; Vernazza 1981; Hirayama 1986) The ionisation ratio $n_{\mathrm{HII}} / n_{\mathrm{HI}}$ usually varies in the interval of 1-3. According to Landman (1984) it is in the range of $0.05-1$, although higher values $(\sim 3)$ have been obtained by Vial (1982).

The prominence magnetic field plays an important role in the QP formation, stability and dynamics. Its magnitude and orientation depend on the FAC flowing within the prominence body. The first measurements of the longitudinal component of the magnetic field (along the prominence axis) have been done by Zirin \& Severny (1961) using the Zeeman effect. The results based on this effect were summarized by Tandberg-Hanssen (1995). With the use of the Hanle effect, a new technique has been developed to measure the longitudinal and transversal prominence magnetic field (Leroy 1985). The absolute magnetic field strength in the QPs is generally found to be in the 
range from a few Gauss to $10 \mathrm{G}$, occasionally reaching 20 or $30 \mathrm{G}$.

Another characteristic of the QPs is the presence of internal motions during their stable period, i.e. when they are not activated. The velocity field of a QP is due to three main types of motions - vertical, horizontal and oscillations. The vertical motions can be divided into two classes - downward flows and upward flows. Downward motions are registered in prominences at the limb (Engvold 1976; Cui Shu et al. 1985) and have values of about $5 \mathrm{~km} \mathrm{~s}^{-1}$. Spectrographic studies (Kubot 1980) give an average value of $0.7 \mathrm{~km} \mathrm{~s}^{-1}$. Upward flows of $0.5-5 \mathrm{~km} \mathrm{~s}^{-1}$ are observed in disk filaments (Mein 1977; Schmieder et al. 1984; Schmieder et al. 1985). Some observations show fast $\left(50 \mathrm{~km} \mathrm{~s}^{-1}\right)$ horizontal motions (inclined to the axis of the prominence at about $20^{\circ}$ ) at the edges of the filaments (Malherbe et al. 1983). Usually, the velocity of horizontal motions in QP is 10 to $20 \mathrm{~km} \mathrm{~s}^{-1}$.

The presence of oscillatory motions in QPs is a proven observational fact (Tsubaki 1989; Vrsnak 1993; Vrsnak \& Ruzdjak 1994). A rough classification of the prominence oscillations, made on the basis of oscillation amplitude, divides them into two main classes; large amplitude (20 $\mathrm{km} \mathrm{s}^{-1}$ ) oscillations affecting the whole object, and small amplitude $\left(3-4 \mathrm{~km} \mathrm{~s}^{-1}\right)$ oscillations affecting the fibril or a restricted area within the prominence (Oliver 1999). The oscillations may have a large range of periods - from a few minutes to hours (Bashkirtsev et al. 1983; Bashkirtsev \& Mashnich 1984; Weihr et al. 1984). The prominence fibril structure also exhibits periodic variations (Tsubaki et al. 1988); individual fibrils may oscillate with their own periods (Thomson \& Schmieder 1991; Yi et al. 1991). There is also some evidence that velocity oscillations are more easily detected at the edges of prominences or where the material seems fainter than in the prominence body (Tsubaki \& Takeuchi 1986; Tsubaki et al. 1988).

In summary, the prominence plasma is a complex system in terms of temperature, density, plasma motion and specific magnetic field configuration. The FAC characteristics are usually unknown and nowadays there are no methods to detect them. The FAC are intrinsically connected with the magnetic field configuration. We suggest that the QP destabilisation can be connected to the FAC changes and the sources of these changes occur at the prominence-chromosphere interface, as is shown in the next section.

\section{Theoretical model}

The MHD wave generation is thought to be among the various processes initiated by the photospheric MHD disturbances, which propagate upward through the QP feet. The MHD wave field-aligned currents (FAC) are supported by Alfven waves. There is another source of FAC, which is primarily due to the Alfven wave gradient existing at the plasma boundaries. In addition to the Alfven modes, the surface waves (SW), supported by these boundaries, can also carry field-aligned currents. The SW FAC should be dislocated, either at the prominence boundaries or within the prominence body. A physical interpretation of SW FAC as a boundary phenomenon, i.e. as FAC flowing into the plasma boundary structures, has also been given (Nenovski 1996). The surface MHD waves have been formerly examined as a source of fieldaligned electric currents and they are suggested to promote QP destabilization via a field-aligned current (FAC) intensification process. The SW can, however, carry FAC only under certain conditions. The SW should be a nonaxial mode, i.e. it has to propagate obliquely to the ambient magnetic field. The SW FAC propagation or the SW group velocity is always directed nearly along the magnetic field.

\subsection{Possible sources of FAC in an arch-type $Q P$}

We have supposed a wave pumping process at the prominence structure feet connected with external sources. These waves are guided into the prominence body through its ends. We have already examined (Madjarska et al. 1996; Nenovski et. al. 1998; Nenovski \& Dermendjiev 1998) a standing global/surface wave structure thought to be imposed on an initial FAC flowing in the prominence body and supporting the QP during its life. The FAC must flow in such a way as to satisfy the requirement of current continuity

$\operatorname{div} j=0$

where $j$ is the current density. We consider the case for which the displacement current can be neglected. One way to determine FAC is to evaluate the divergence of the parallel component of the current density $\boldsymbol{j}_{\|}$. Since the charge separation is negligible in a large scale plasma, i.e. div $\boldsymbol{j}=0$, we can relate this current density $\boldsymbol{j}_{\|}$to the corresponding perpendicular one $\boldsymbol{j}_{\perp}$ by the equation

$\boldsymbol{j}_{\|}=-\nabla \cdot \boldsymbol{J}_{\perp}$

where $\boldsymbol{J}_{\perp}$ is the height-integrated perpendicular current density $j_{\perp}$, i.e. the currents flowing within the whole region of the prominence foot near the chromosphere. In this region there are Pedersen and Hall parts of the $\boldsymbol{J}_{\perp}$ current. This equation expresses the current balance condition at the chromosphere level, i.e. the term $-\nabla \cdot \boldsymbol{J}_{\perp}$ acts as a source of a parallel current, which could communicate between adjacent regions in the QP body. In this way the plasma of the upper part of the QP can be coupled electro-dynamically with the plasma in the prominence feet, or the chromosphere.

\subsection{Reflection conditions for MHD surface waves at the QP feet}

We examine more concretely in this work a FAC intensification process by surface/global modes guided in a arch-shaped prominence structure. Such a process will be 
possible if an SW-mode propagating along the arch QP structure undergoes multiple reflection. It is quite natural to suppose that when such a wave mode meets the boundary (the prominence feet or the chromosphere) it may be transformed and/or reflected. Alfven and MHD surface modes carry field-aligned current that should be closed at this boundary. The wave energy flux could be absorbed or reflected at the boundary, depending on the matching impedance between the prominence body on one hand, and the chromosphere, on the other hand. Therefore, it is very important to study the reflection conditions for MHD surface waves at the QP feet.

The reflection coefficient for an Alfven wave mode propagating in the prominence and impinging the chromosphere region from above can be given by (Scholer 1970)

$R=\frac{\sum(A)-\sum(P)}{\sum(A)+\sum(P)}$

where $\sum(P)$ and $\sum(A)$ are the height-integrated Pedersen conductivity and wave (Alfven) conductance, respectively. The reflection coefficient $R$ expresses a juxtaposition of the high-integrated Pedersen conductivity and the Alfven wave conductance at the reflection point (volume) where the FAC is closed. Scholer's examination (1970) of the reflection condition considers the Alfven wave velocity at the point of reflection of incident wave. This Alfven velocity magnitude suffices for the wave conductance determination.

The expression for $R$ is derived under condition (1) treated above. Physically, it means that the Alfven wave field-aligned current is completely closed by the perpendicular Pedersen and Hall currents flowing through the chromosphere region. Hence, if the reflection coefficient $R$ is positive and, for example, $R \longrightarrow 1$, the $\mathrm{FAC}$ of the incident and reflected Alfven waves cancel each other because $E_{\uparrow}=R E_{\downarrow}$, where $E_{\downarrow}$ and $E_{\uparrow}$ are the electric fields of the incident and reflected Alfven waves, respectively. The wave magnetic field

$b=b_{\downarrow}+b_{\uparrow}=\frac{e_{\varphi}}{V_{\mathrm{A}}}\left(E_{\downarrow}-R E_{\downarrow}\right) \simeq 0$

and the corresponding velocity will be relatively small. Therefore, the FAC cannot be intensified and the FAC from the source stays constant. Moreover, because of the wave damping mechanisms, the FAC will diminish its amplitude at each reflection event and will go to zero. On the other hand the internal resistance $1 / \sum(P)$ can be considered as infinite and the FAC of the incident Alfven wave should be completely blocked from closure.

Inversely, if the reflection coefficient $R$ is negative, the wave magnetic field and the corresponding velocity will be increased and the Alfven wave FAC could be intensified. At $R=-1$ the Alfven wave electric field will be reflected out-of-phase. However, the wave magnetic field

$b=\frac{e_{\varphi}}{V_{\mathrm{A}}}\left(E_{\downarrow}-R E_{\downarrow}\right)=2 \frac{e_{\varphi}}{V_{\mathrm{A}}} E_{\downarrow}$

will be reflected in phase and in this way will be doubled.
Generally, the reflection alters the amplitudes of the wave field and plasma velocity. In the case $R<0$ the wave magnetic energy density increases after reflection by a factor of $(1-R)^{2}$, which under the condition $R \rightarrow-1$ amounts to nearly 4 . The plasma velocity $u=E \times \frac{B_{0}}{B_{0}^{2}}$, however, reduces its value. An increase in the wave magnetic field magnitude results in an enhancement of the field-aligned current $j_{\|}$. In a detailed treatment, the wave absorption has to be taken into account. We examine it in paragraph 3 . This could additionally reflect on the phase difference at the point of reflection but the effect of the reflection processes on the wave field and velocity amplitudes remains.

Also, at $R=-1$ the resistance $1 / \sum(P) \sim 0$ and the FAC is completely closed across the chromosphere through the source $-\nabla \cdot \boldsymbol{J}_{\perp}$ This should lead to FAC intensification process at each reflection event.

The reflection coefficient $R$ (Eq. (3)) is assumed to be the same for a MHD surface wave mode. In both cases (Alfven and MHD surface waves) the FAC propagates with the Alfven velocity along the ambient magnetic field. The MHD surface wave and the wave FAC being launched reach the appropriate prominence foot to be transformed. We examine the chromosphere influences on such wave structures.

To study the reflection conditions for MHD surface waves at the QP feet we construct a one-dimensional model of a prominence foot with magnetic field, neutral and plasma density and temperature distributions depending only on height $(z$-coordinate). The computational grid covers an area of $1.5 \times 10^{3} \mathrm{~km}$ with step $\Delta=30 \mathrm{~km}$. The initial values of the prominence plasma parameters and magnetic field are varied to simulate different initial conditions at the prominence feet. These variations depart from the QP standard model (Jensen \& Wiik 1990) and the average network chromosphere (model D) (Vernazza et al. 1981). Next, for every given initial condition we compute the step-integrated Pedersen $\sigma_{\perp}(z)$ and Alfven wave $1 /\left(\mu_{0} V_{\mathrm{A}}(z)\right.$ conductivity, where $\sigma_{\perp}$ is the conductivity perpendicular to the magnetic field lines, and $\mu_{0}$ is the magnetic permeability. The conductivity $\sigma_{\perp}$ consists of two terms: $\sigma_{\perp, \mathrm{i}}$ due to the ions and $\sigma_{\perp, \mathrm{e}}$ due to the electrons, which are expressed by formulae

$$
\begin{aligned}
& \sigma_{\perp, \mathrm{e}}=\frac{N e^{2}}{m_{\mathrm{e}}} \frac{\nu(e, H)}{\nu(e, H)^{2}+\omega_{\mathrm{ce}}^{2}}=\frac{N e^{2}}{m_{\mathrm{e}}} \frac{\nu_{\mathrm{en}}}{\nu_{\mathrm{en}}^{2}+\omega_{\mathrm{ce}}^{2}} \\
& \sigma_{\perp, \mathrm{i}}=\frac{N e^{2}}{m_{\mathrm{e}}} \frac{\nu\left(H^{+}, H\right)}{\nu\left(H^{+}, H\right)^{2}+\omega_{\mathrm{ci}}^{2}}=\frac{N e^{2}}{m_{\mathrm{e}}} \frac{\nu_{\mathrm{in}}}{\nu_{\mathrm{in}}^{2}+\omega_{\mathrm{ci}}^{2}}
\end{aligned}
$$

assuming an hydrogen plasma only (Banks 1966).

Here $e$ is the electron charge, $m_{\mathrm{e}}$ and $m_{\mathrm{p}}$ are the electron and proton mass, respectively $\nu_{\mathrm{en}}$ is the frequency of electron-neutral collisions $\nu_{\text {in }}$ is the ion-neutral collision frequency, $B$ the magnetic field and $\omega_{\mathrm{ce}}=e B / m_{\mathrm{e}}$ and $\omega_{\mathrm{ci}}=e B / m_{\mathrm{H}}$ are cyclotron electron and ion frequencies, respectively. 

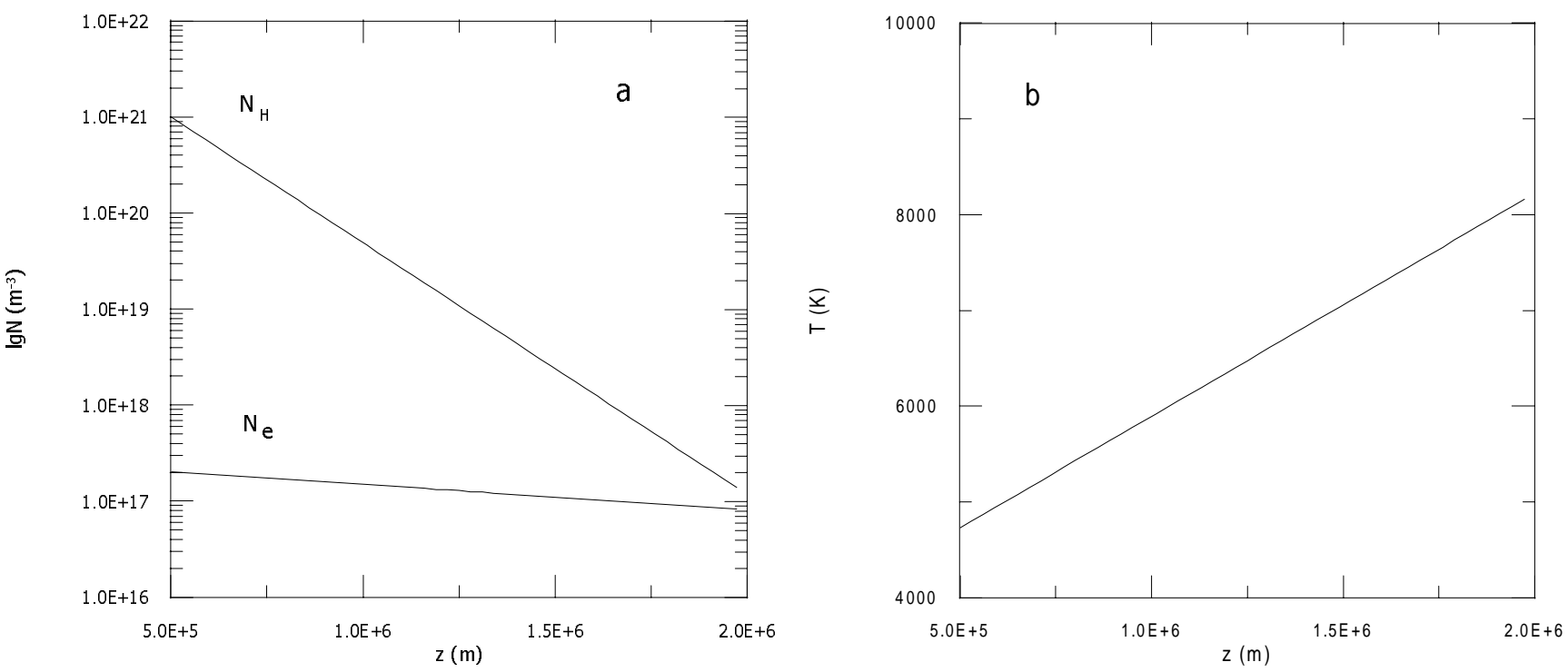

Fig. 1. The electron $N_{\mathrm{e}}$ and hydrogen $N_{\mathrm{H}}$ density a), and temperature b) profiles with altitude taken as an initial plasma condition in the prominence foot.

The corresponding collision and cyclotron frequencies are computed by formulas (Priest 1981; Cowling 1976)

$\nu_{\mathrm{en}}=\frac{1}{\tau_{\mathrm{en}}}, \nu_{\mathrm{in}}=\frac{1}{\tau_{\mathrm{in}}}$

where

$\tau_{\mathrm{en}}=\frac{\tau_{\mathrm{ei}} T^{2}}{5.2 \times 10^{-12}} \frac{N_{\mathrm{n}}}{N_{\mathrm{e}}}, \tau_{\mathrm{ei}}=0.266 \times 10^{5} \frac{T^{\frac{3}{2}}}{N_{\mathrm{e}}}$

$\tau_{\text {in }}=\tau_{\text {en }} \frac{\omega_{\mathrm{ce}}}{2 \omega_{\mathrm{ci}}}\left(\frac{m_{\mathrm{e}}}{m_{\mathrm{p}}}\right)^{\frac{1}{2}}$.

Here, $\tau_{\mathrm{ei}}$ is the electron-ion collision time interval, $\tau_{\mathrm{en}}$ and $\tau_{\text {in }}$ are the corresponding collision times for neutrals with electrons and ions.

In the next step, we compute a height-integrated conductivity introduced in the following way. First, at every grid step we compute $\sigma_{\perp}=\sigma_{\perp, \mathrm{i}}+\sigma_{\perp, \mathrm{e}}$ and verify whether the inequality $\nu_{\mathrm{in}} \geq \omega_{\mathrm{ci}}$ is fulfilled or not. The equality $\nu_{\text {in }}=\omega_{\text {ci }}$ marks the maximum $z$ (height) of the conductivity layers where the FAC can be closed through the prominence foot (at this height the Pedersen conductivity $\sigma_{\perp}$ has a maximum). Next, we sum all step-integrated conductivities for which the inequality $\nu_{\text {in }} \geq \omega_{\text {ci }}$ is fulfilled. This cumulative height-integrated conductivity is given by

$\sum(P)=\sum \sigma_{\perp}(z)$

Looking for the reflection conditions, we compute the local Alfven velocity/wave conductance for every computational grid step, checking the ratio between local Alfven wave conductance and height-integrated Pedersen conductivity. What we really consider is the local Alfven velocity/conductance at the height of closure of the fieldaligned current, i.e. at the height of the maximum of the Pedersen conductivity.
The main aim is to find the situations under which the FAC intensification process can take place. According to (3) the Pedersen conductance should be compared with the wave (Alfven) conductance

$\sum(A)=\frac{1}{\mu_{0} V_{\mathrm{A}}(z)}$.

Such a situation involves an appropriate combination of magnetic field strength $B$ and neutral, $N_{\mathrm{n}}$, and plasma, $N$, densities.

The computational results show that, under standard prominence plasma and magnetic fields conditions (Jensen \& Wiik 1990), the inequality $\nu_{\text {in }} \geq \omega_{\text {ci }}$ is not fulfilled and the refraction coefficient $R$ is positive.

The reflection coefficient $R$ only attains negative values when we simulate an intense plasma density enhancement in prominence feet. In our calculations, we assume, as initial conditions in the prominence foot, plasma parameters corresponding to the VAL network chromosphere model of Vernazza et al. (1981).

These computations were done in the following way. Using the atmospheric parameters $\left(T, N_{\mathrm{e}}, N_{\mathrm{h}}\right)$ for the average network chromosphere (model D) (Vernazza et al. 1981) we compute linear regression approximation for the hydrogen number density

$\log N_{\mathrm{H}}(z)=22.3-0.262 \times 10^{-5} z$

the electron number density

$\log N_{\mathrm{e}}(z)=17.4-0.265 \times 10^{-6} z$

and the temperature

$T(z)=0.357 \times 10^{4}+0.233 \times 10^{-2} z$.

We compute the magnetic field $B$ by the formula

$B(z)=B_{0} \exp ^{-z / 2 H_{\mathrm{B}}}$ 


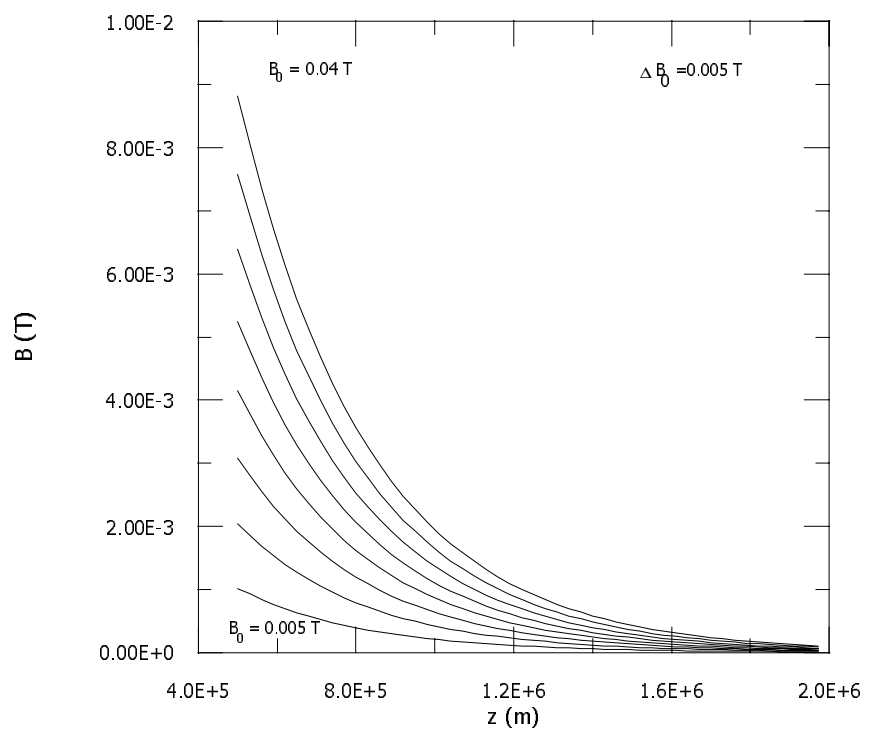

Fig. 2. The magnetic field profile with altitude in the prominence foot, computed for different values of the magnetic field $B_{0}$ at photospheric level.

where,

$H_{\mathrm{B}}=\frac{P_{0}+\frac{B_{0}^{2}}{2 \mu_{0}}}{\rho_{0} g}$

is the magnetically modified scale height, and $\rho_{0}$ and $P_{0}$ are the respective model values of the density and the pressure at height $h=0$ corresponding to the photospheric level. Here the basic parameter is $B_{0}$, the magnetic field at photosphere level, which we vary from $B_{0}=0.04 T$ to $B_{0}=0.0025 T$ by steps of $\Delta B_{0}=0.0025 T$.

Using the Eqs. (13)-(17) we simulate the conditions in a QP foot corresponding to the VAL model D for heights starting at $h=500 \mathrm{~km}$ and ending at $2000 \mathrm{~km}$. The profiles of the plasma parameters $\left(T, N_{\mathrm{e}}, N_{\mathrm{h}}\right.$ and $\left.B\right)$ are shown in Figs. 1 and 2.

To trace the maximum height of the conductive layers where the FAC starts to be closed, we construct the quantity $f(z)=\nu_{\text {in }}(z)-\omega_{\text {ce }}(z)$ as a function of the $z$ coordinate. The profiles of $f(z)$ for 8 values of $B_{0}$, from $B_{0}=0.04 T$ to $B_{0}=0.005 T$ by steps of $\Delta B_{0}=0.005 T$, are shown in Fig. 3. It is clearly seen that $f(z)$ becomes positive for values of $B_{0}$ less than $0.0275 T$. One can trace the height where $f(z)$ jumps from negative to positive values and evaluate the height $h$ above the lower boundary (the bottom of the prominence foot) where FAC will be closed. For example, in the case of $B_{0}=0.0225 T$, which corresponds to $B(z=500)=0.0047 T$ and $B_{0}=0.0075 T$, which corresponds to $B(z=500)=0.0017 T$, this will occur at $h=635 \mathrm{~km}$ and $h=1055 \mathrm{~km}$, respectively. The dependence of $h$ on the magnetic field $B_{0}$ is shown in Fig. 4.

In the next step, we compute the corresponding cumulative height-integrated conductivities $\sum(P)$ and $\sum(A)$ for $z \leq h$ to determine the reflection coefficient $R$ as a function of $B_{0}$. The results obtained are given in Figs. 5

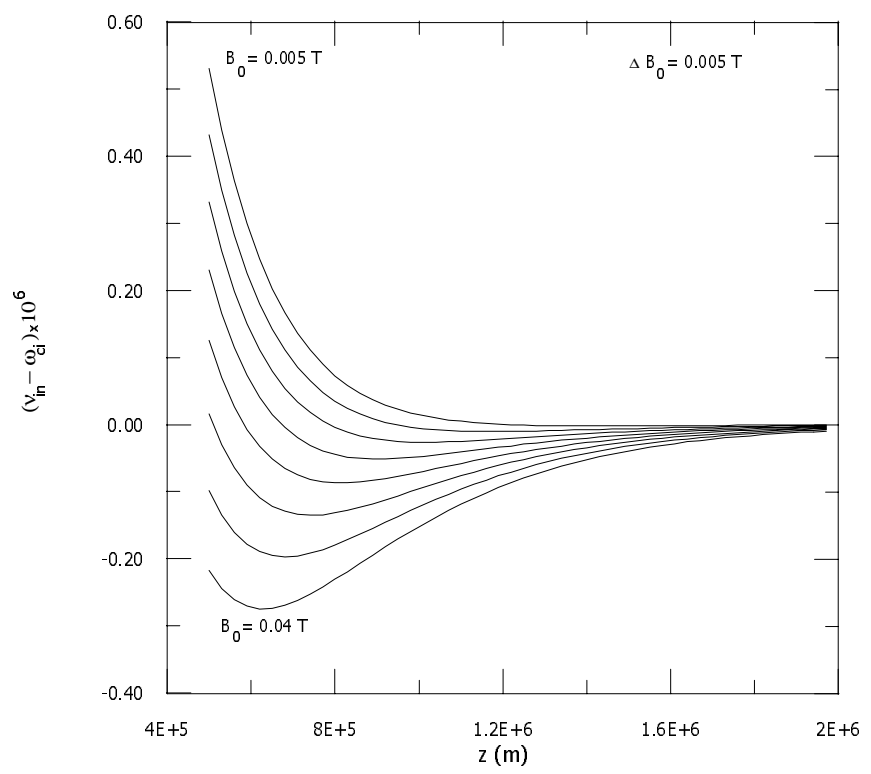

Fig. 3. The altitude profiles of $f(z)=\nu_{\mathrm{ni}}(z)-\omega_{\mathrm{ci}}(z)$ for different $B_{0}$.

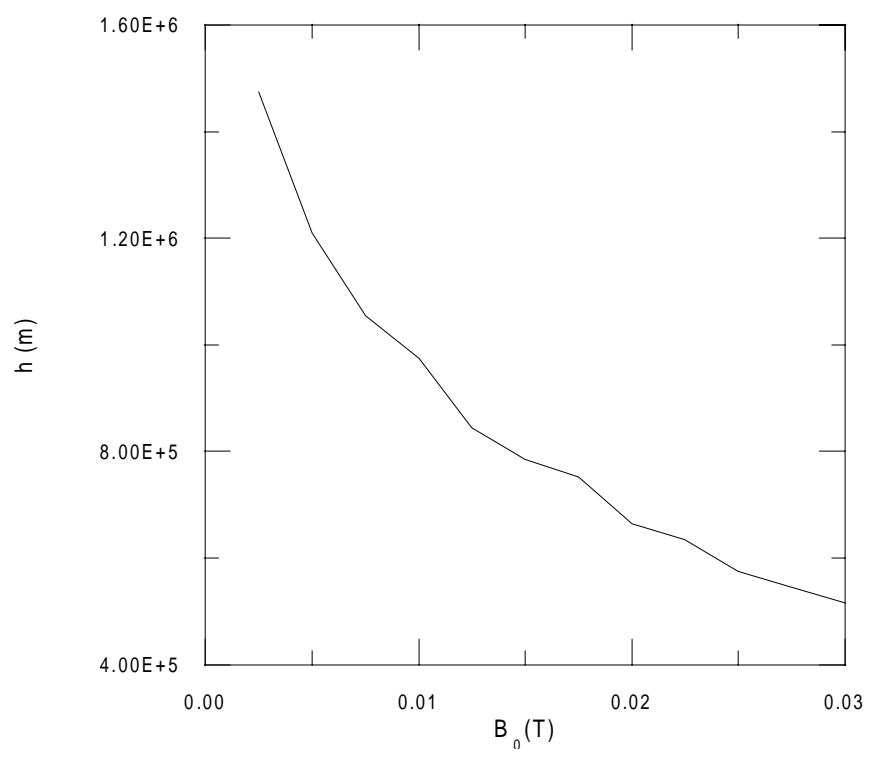

Fig. 4. Dependence of the height, where the Pedersen current will start to be closed, on the magnetic field $B_{0}$ at photospheric level.

and 6. It is well seen in these figures that $\sum(P) \geq \sum(A)$ and $R \leq 0$ for $B_{0} \geq 0.0125 T$.

\section{The MHD wave dissipation length}

Normally, the wave FAC intensification rate at each reflection event (if it takes place) is compensated by the SW dissipation rate along the prominence body. The SW dissipation may be expressed by a characteristic damping length $L(A)$. Under prominence conditions, the surface mode dissipation losses are dominated by collision 


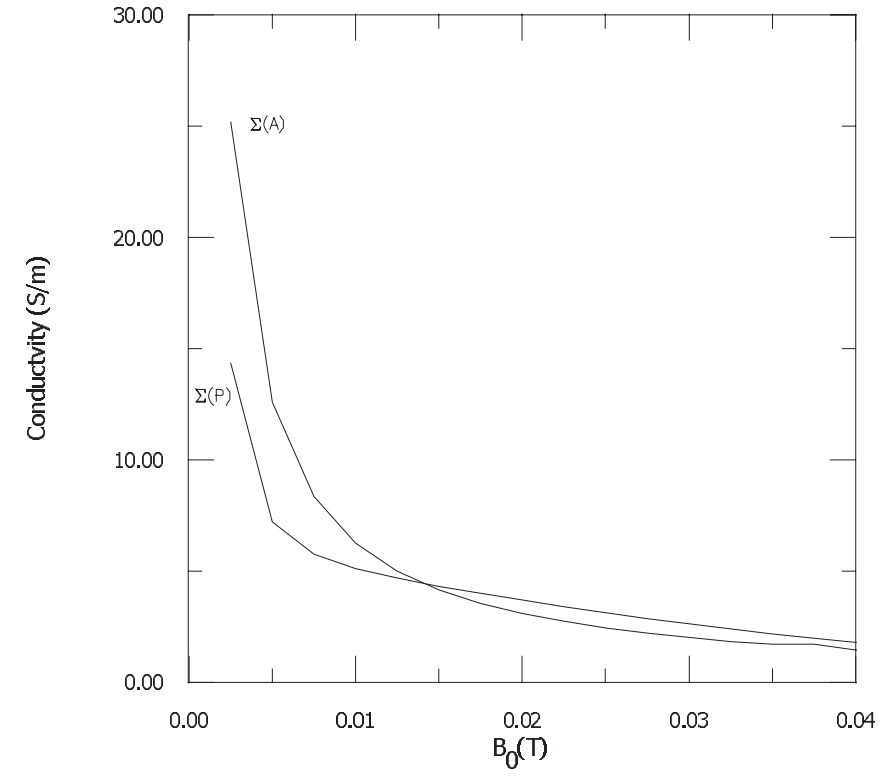

Fig. 5. The cumulative height-integrated Pedersen $\sum(P)$ and wave $\sum(A)$ conductivity as a function of $B_{0}$.

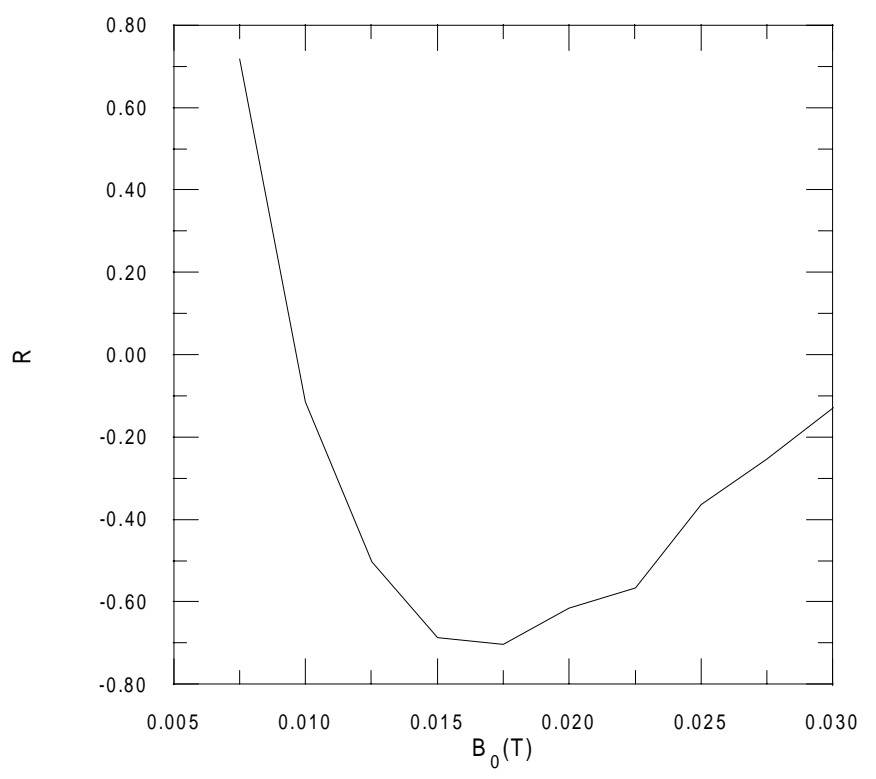

Fig. 6. The reflection coefficient $R$ as a function of $B_{0}$.

processes. Hence, we can represent $L(A)$ explicitly by the ratio

$L(A)=\langle S\rangle / Q$

where $Q$ is the volumetric damping/heating rate and $\langle S\rangle$ is the time-averaged $z$-component (along the FAC direction) of the Poynting flux. The $z$-component of the Poynting vector $\langle S\rangle$ is evaluated in the MHD approach by

$\langle S\rangle=(2 \tau(A))^{-1} \operatorname{Re} \int \mathrm{d} t\left(p V^{*}+b \times\left(V^{*} \times B\right)\right) \cdot \hat{z}$

where $p$ is the total (magnetic + gas) pressure, $\tau(A)$ is the SW mode period and $b$ and $V$ are the MHD wave magnetic field and velocity, respectively. By using the normal mode analysis for the compressive MHD wave we obtain:

$\langle S\rangle=2 \pi \rho\left[c_{\mathrm{s}}^{2}+V_{\mathrm{A}}^{2}\right]^{2} k_{z}(\nabla \cdot V)^{2} \tau^{3}(A)$

where $\rho$ is the plasma density, $c_{\mathrm{S}}$ is the sound velocity and $\omega=2 \pi / \tau(A)$ and $k_{z}$ are frequency and wave number component along the prominence flux tube, respectively.

The MHD wave dissipation depends mainly on viscosity and conductivity. Let us evaluate quantitatively either mechanism. Under the condition $\omega_{\mathrm{ci}} \geq \nu_{\text {in }}$ that is fulfilled for the prominence body, the volumetric heating rate can be expressed by

$Q=\eta / 3(\nabla \cdot V)^{2}$,

where $\eta$ stands for the viscosity. Concerning the relationship between viscosity $\eta$ and pressure $P$ an expression exists:

$\eta=\frac{P}{\nu_{\text {in }}}$

(An analogous expression for fully ionized plasma is used by Shkarofsky et al. 1996.) We illustrate a more frequent situation applicable in the solar atmosphere. The viscosity mechanism is dominant under high conductivity conditions. Hence, for the MHD wave damping length $L(A)$ we obtain

$L(A)=(2 \pi)^{-2} \rho \frac{L^{3}}{\tau(A) \eta}$

where $L$ is the prominence arch length.

Inversely, if the conductivity is sufficiently low, the viscosity dissipation mechanism can be less effective than the dissipation produced by the conductivity itself. According to the MHD wave theory, the damping rate $\nu$ of the Alfven/magnetosonic waves can be expressed (Boyd \& Sanderson 1969) by

$\nu \equiv-k^{2} / \mu_{0} \sigma$

where $k$ stands for the parallel (for Alfven waves) or total (for the magnetosonic waves) wavenumber. For our study, the conductivity $\sigma$ is assumed to be identical to the Pedersen one. Depending on the spatial scales (mainly in the direction perpendicular to the ambient magnetic field), either dissipation mechanism acts effectively. Considering transverse scales much smaller than the axial one, i.e. $k_{\|} \ll k_{\perp}$, we estimate that the dissipation mechanism due to the low conductivity is prevailing. The corresponding MHD wave damping length $L(A)$ is given by

$L(A)=L a^{2} \sum(P) \mu_{0} / \tau(A) h$

where $a$ is the magnetic tube radius. We note that in both cases the SW damping length $L(A)$ is sensitive to the SW period $\tau$, the magnetic flux tube radius $a$ and the neutral density. The wave period $\tau(A)$ decreases when the plasma density $N_{\mathrm{e}}$ decreases. Then the dissipation length 


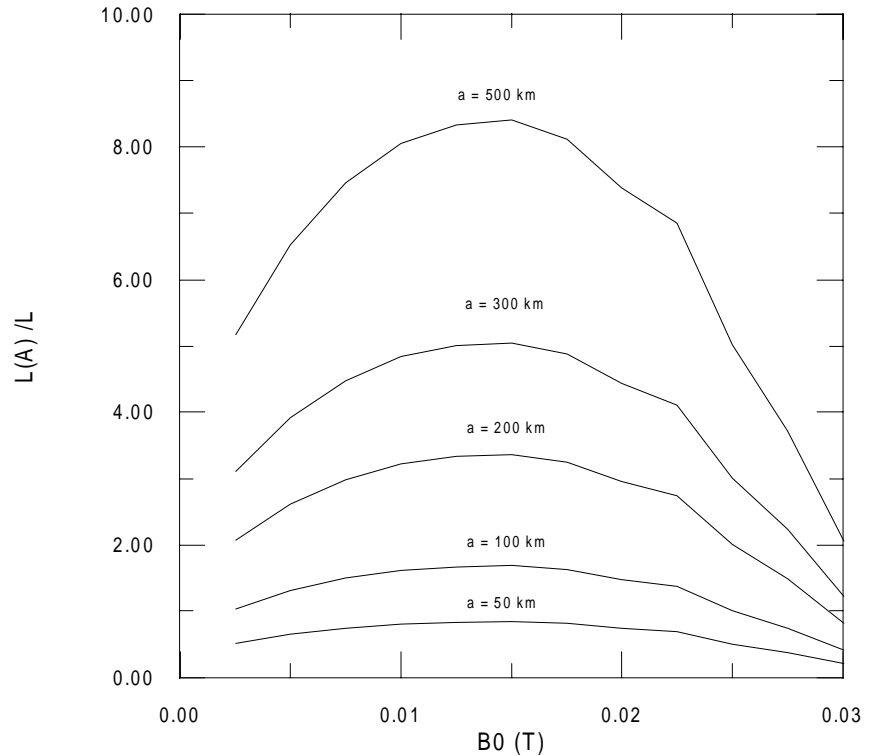

Fig. 7. Dependence of the ratio of the MHD wave damping rate length $L(A)$ and the prominence length $L$, on the magnetic field $B_{0}$ at photospheric level for different flux tube radii $a$.

increases as follows from (23) and (25). This dependence should be further taken into account in our analysis.

In general, the net FAC intensification rate directly depends on the time $\tau$, needed for FAC to propagate along the magnetic flux tube, given by

$\tau=2 \int \sqrt{g} \mathrm{~d} s / V_{\mathrm{A}}$

Here $g$ is the diagonal component of an appropriate metric tensor (accounting for the geometry). We note also that unlike the local Alfven wave resonances, the SW period does not depend on the magnetic field line location coordinate. However, it depends on the large-scale magnetic field and density gradients along the magnetic field tube. These properties do not prevent formation of a stable FAC structure.

We have performed a calculation of the $L(A) / L$ ratio under the given plasma chromosphere and prominence conditions. The estimate of this ratio gives the dominant role to the low conductance mechanism on the MHD wave dissipation. The viscous mechanism would be essential for the local small-scale wave structures and their fieldaligned currents. In our case of an MHD surface wave, the dominant mechanism is due to the low-conductivity chromosphere. This situation appears to be analogous to the ultra-low-frequency (ULF) wave dissipation in the Earth magnetosphere (Newton et al. 1978). The dissipation of the ULF waves is determined mainly by the ionospheric Pedersen conductivity. In our case with the MHD wave mode in the prominence/chromosphere system, the $L(A) / L$ ratio depends on the ambient magnetic field magnitude and the flux tube radius. This dependence is illustrated in Fig. 7. It should be mentioned that for a thin flux tube with radius of $50 \mathrm{~km}$ this ratio is close to 1, i.e. the effective wave dissipation length is comparable to the prominence length $L$.

The physical meaning of these results is the following. Under the usual quiet conditions the MHD wave cannot be amplified, i.e. it cannot be a source of prominence destabilisation. The wave energy dissipation overwhelms the energy gain due to any instability mechanism. However, the situation is reversed when we have an enhancement of the chromosphere conductivity. Then there are two mechanisms acting on the MHD wave magnitude and its FAC. The wave energy dissipation decreases, its dissipation length is enlarged and the wave reflection condition switches on the wave FAC enhancement. The later requires a higher Pedersen conductivity in comparison to the wave (Alfven) conductivity. The interplay between both processes can be quantitatively expressed by the following balance principle consideration.

From the balance of the input energy (the FAC enhancement process) due to the reflection process at the chromosphere $W_{\text {chrom }}(R)$ and the dissipated wave energy $W_{\text {loss }}(\omega, k)$ (due to the dissipative losses of the wave $(24)$ ), which requires

$W_{\text {chrom }}(R)=W_{\text {loss }}(\omega, k)$,

we find the threshold condition of the FAC instability in the QP system. Usually $W_{\text {chrom }}(R)$ is negative and FAC instability is impossible. Under higher chromosphere conductivity conditions $W_{\text {chrom }}(R)$ becomes positive and an energy (FAC) gain is possible. Let us focus our attention to the mechanism of FAC feeding at the chromosphere and the wave magnetic field decrease in the QP body.

First we notice that if the dissipative losses were absent, the input energy $W_{\text {chrom }}(R), R \leq 0$ would wholly go to the wave field energy increase and consequently to the FAC enhancement supported by this wave. The latter may be expressed (in a linear approximation) as dissipative losses with a negative damping rate, i.e. the SW field would increase with a growth rate $\gamma$ determined by the equality

$$
\begin{aligned}
W_{\text {chrom }}(R) & \equiv(1-R)^{2} b_{\omega, 0}^{2} / 2 \mu_{0} \\
& =-W_{\text {loss }}\left(R \nu_{\mathrm{A}} / L\right) \\
& \equiv\left(b_{\omega, 0}^{2} / 2 \mu_{0}\right) \exp \left[\left(2 L / \nu_{\mathrm{A}}\right) \gamma\right]
\end{aligned}
$$

where $\gamma$ stands for the negative damping rate. For a dissipative medium, such as the QP plasma, the SW damping $W_{\text {loss }}$ is determined by the effective damping length $L(A)$, or the damping frequency $\nu$ (determined through $\left.\nu=\nu_{\mathrm{A}} / L(A)\right)$. Then a certain part $W_{\text {loss }, 1}$ goes to cancel the SW magnetic field increase in the chromosphere. According to the above-mentioned energy balance treatment, the energy $W_{\text {loss }, 1}$ may be expressed as that of the input wave energy gain increasing with a growth rate $\left(\nu_{\mathrm{A}} / L\right) \ln (1-R)$. Hence the real damping depends on $\gamma-\nu=\left(\nu_{\mathrm{A}} / L\right) \ln (1-R)-\nu_{\mathrm{A}} / L(A)$ and the Eq. (28) becomes

$$
\begin{aligned}
W_{\text {loss }, \text { new }} & =W_{\text {loss }}-W_{\text {loss }, 1} \\
& =W_{\text {loss }}\left[\left(\nu_{\mathrm{A}} / L\right) \ln (1-R)-\nu_{\mathrm{A}} / L(A)\right],
\end{aligned}
$$


where usually $W_{\text {loss,new }} \leq 0$. When the reflection coefficient $R$ becomes negative, both competitive processes the surface wave FAC intensification rate at the chromosphere and the SW dissipation rate - should determine the following threshold condition for a net FAC enhancement: $W_{\text {loss,new }}=0$.

Therefore the FAC instability will start at

$$
(L(A) / L) \ln (1-R) \geq 1
$$

The inequality (30) yields the condition for a net FAC enhancement in the prominence plasma and depends on the plasma parameter conditions entering in both parameters $R$ and $L(A)$.

Under the assumption of an external wave source supplying wave energy in the prominence structure, two ultimate situations are possible: 1) $R>0$, the wave magnetic field energy density decreases in the process of reflection and, subsequently, the surface/Alfven wave damping, mainly due to dissipative losses; 2) $R<0$, the wave magnetic field energy density increases in the process of reflection, and the wave $\mathrm{FAC}$ increases, too. Under this condition, a compensation of dissipative losses is allowed. That is why the process of loss compensation requires a threshold feature described by formula (30) in our paper. Once again we emphasize that: 1) We study the conditions under which the mechanism of magnetic field energy density increase/FAC enhancement at the chromosphere/prominence boundary is switched on; 2) The energy supply of this instability is supposed to be connected with an external source of such waves. Similar process of FAC enhancement has been studied by Murata (1991). An Alfven wave FAC enhancement process in a magnetic arcade with shear motion at the foot point has been examined. He did not, however, consider the real dissipative losses in the system.

Examining condition (30) we see that it is usually fulfilled for $L(A) \geq L$, i.e. the dissipation length should be sufficiently great. Since $L(A)$ is inversely proportional to the wave period $\tau$, we may conclude that the wave dissipation decreases when the period $\tau$ decreases, i.e. greater $L(A)$ requires a lower density $\mathrm{Ne}$ and/or greater magnetic field $B$ in the prominence body (see 26).

Since the reflection coefficient $R$ should be negative, a higher plasma density in the chromosphere is needed as well. Therefore, the suggested mechanism of surface wave FAC intensification could be switched on under: a) higher plasma densities at the chromosphere-prominence intersection points, and b) lower plasma density conditions in the prominence body. The first condition enables an increase of the Pedersen conductivity and results in greater dissipation lengths $L(A)$; the second condition results in a decrease of the wave conductivity. The fulfillment of both conditions are necessary for the wave field-aligned current enhancement. Since the FAC intensification in the prominence body is forced, a destabilisation process is started and ultimately, QP magnetic field destruction could be produced.

\section{Implications for prominence destabilisation}

The basic relations obtained in Sect. 2.3 yield some criteria for a QP destabilisation. The inequality (30) is crucial. It is obvious that when $R>0$ there is no way to get FAC enhancement. Therefore $R$ should be near -1 in order to expect a destabilising effect. As we have seen in Sect. 2.1. under conditions corresponding to the VAL network chromosphere model in the prominence foot, the reflection coefficient $R$ can easily obtain a negative value. Then the logarithm is positive and equal to 0.7 at $R=-1$. Hence the critical value for a FAC initialisation is determined by

$L(A) / L \geq 1.4$.

Under such conditions, the destabilisation can be switched on due to sudden changes in the plasma and neutral densities implicitly entering in (31). The allowed neutral density changes in the QP structure, however, have to be lower than a certain value, critical for MHD wave existence. Under the ion collision damping conditions $\left(\nu_{\mathrm{in}}<\omega_{\mathrm{ci}}\right)$, the required upper limit for the neutral density-magnetic field ratio is

$N_{\mathrm{n}} / B \leq \alpha 10^{23}$

in SI units, where $\alpha$ is a constant of the order $\mathrm{O}(1)$. This inequality should be fulfilled in the QP structure above the prominence feet.

The FAC closing in the low-altitude region only requires an additional restriction on the density and the magnetic field variations. Under the conditions $\nu_{\text {in }}>\omega_{\text {ci }}$ and $R<0$ it is given roughly by

$N_{\mathrm{n}} \sqrt{N} / B<1.5 \times 10^{32}$

where the magnetic field $B$ and neutral and plasma densities $N_{\mathrm{n}}$ and $N$ are mean values for the prominence body. In the case when the wavenumber $k$ coincides with the parallel wavenumber $k_{\|} \approx 2 \pi / n L(n=1,2 \ldots)$, with axial SW modes, inequality (31) is prevented from being fulfilled unless the arch length $L$ is much smaller, as in the case of a small arch-fibril. For the case of the SW kink, the azimuthal wavenumber $k_{\perp}$ exceeds the parallel wavenumber and inequality (31) may easily be satisfied for larger QP structures.

In general, (25) and (31) yield all the principal requirements for FAC intensification by a bouncing SW mechanism and a possible QP destabilisation.

Let us now make a numerical estimate of these parameter requirements. If we take for the plasma neutral density ratio $N / N_{\mathrm{H}}$ an observational value, i.e. equal to 3 , from (32) we derive

$N \leq 1.5 \times 10^{13} \times\left(B / B_{0}\right)^{2}$

where a prominence foot value of $10^{2} \mathrm{G}$ is taken for $B_{0}$. At mean value $B=10 \mathrm{G}$ the plasma density does not exceed $10^{9} \mathrm{~cm}^{-3}$, which is a reasonable value. Further, when we put a base plasma density equal to $N_{0}=10^{15} \mathrm{~cm}^{-3}$ 
and the base ion-cyclotron frequency $\omega_{0}$ equal to $10^{6} \mathrm{~s}^{-1}$ in (31) we obtain

$$
\left(a / a_{0}\right)^{2} \sum(P) \geq 1.6 \ln (1-R)\left(h / h_{0}\right) \tau(A)
$$

where the prominence radius $a$ and the height of the FAC closure $h$ are normalized quantities. Here $a_{0}=500 \mathrm{~km}$ and $h_{0}=500 \mathrm{~km}$. For $a \cong 500 \mathrm{~km}, h \cong 103 \mathrm{~km}, \sum(P)=$ $10 S$ and $\tau(A) \cong 10^{3} \mathrm{~s}$ the above inequality cannot be fulfilled. In the case of greater prominence radii, shorter wave periods, e.g. $a \geq 1500 \mathrm{~km}$ and $\tau(A) \leq 600 \mathrm{~s}$, the above criterion could be fulfilled under real chromosphere conductance condition. The latter should be of order of $\sum(P) \geq 4-5 S$. Hence, the principal obstacle for such a wave mechanism is an exact evaluation of the dissipation and the corresponding dissipation length $L(A)$ needed for the estimates of the required prominence magnetic fields and plasma densities.

The above considerations suggest a scenario of FAC intensification and possibly QP destabilisation. The FAC intensification mechanism by a MHD surface wave can be switched on under lower plasma density conditions in the prominence body and higher plasma densities at the prominence footpoints. This situation guarantees the FAC closing and feeding via chromosphere currents. Thus the chromosphere electric currents may be shunted through the prominence body. Consequently the FAC increase in the prominence body causes an additional destabilising force and a condition for QP magnetic field destruction can be met. The duration of the FAC intensification process depends mainly on the local value of the reflection coefficient $R$, the neutral plasma density ratio, the QP length, and the magnetic strength.

\section{Conclusion}

We propose here a possible way of generating FAC in an arch-type QP by MHD surface wave modes propagating across or oblique to the prominence magnetic field. These "wave induced" currents tend to be concentrated at the prominence periphery, i.e. they are surface field-aligned currents. We show that such currents can be intensified in the case when surface wave bouncing processes at the prominence feet are possible. The standing wave mechanism of FAC intensification has been already applied to the Earth magnetosphere conditions. The new viewpoint in our model is the inclusion of SW or boundary modes as FAC carriers. This surface FAC yields an increase of the azimuthal component of the magnetic field, which in turn may destabilise the whole QP system.

Our parameter evaluation accounts for simultaneous density and magnetic field variations both at the QP footpoints and the prominence body. The preferable situations for such a "switch on" of QP destabilisation process requires a plasma density increase at the footpoints and/or a low density plasma condition in the prominence body. Therefore, the magnetic field strength requirements depend on the neutral density variations and the neutral-plasma density ratio. We show, as well, that the FAC reinforcement could provoke a destabilization of the whole QP structure. The destabilization process depends quantitatively on both the FAC intensification rate and wave dissipation. In Sect. 3 of our work we gave some estimations of the wave magnetic field damping rate resulting from the cross-field electric conductivity in partially ionized plasma, as well as its damping rate. The obtained damping rates determine the threshold condition for FAC intensification. In this way we take into account the effects of electric conductivity.

Our results refer to a simplified model of prominence structure. We consider a FAC intensification mechanism due to an active chromosphere process - the plasma density and conductivity enhancement. Detailed examinations of all the plasma parameters in the prominence body are further needed. The obtained criteria for destabilization are suitable for observational verification of the proposed mechanism of FAC generation. As well, data from SOHO/SUMER, CDS, EIT could contribute to the verification of the proposed scenario for QP destabilization.

Acknowledgements. The authors thank the referee for his useful comments. This research was partially supported by the National Scientific Fund (Bulgaria) under Grant 815/98. V. D. would like to thank to prof. I. Zhelyaskov for the helpful discussions. K.B. and J.-C. V. acknowledge the support of the PECO/NEI (project 5135) in the frame of the "Coopération Franco-Bulgare".

\section{References}

Banks, P. 1966, Planet Space Sci., 14, 1105

Bashkirtsev, V., Kobanov, N., \& Mashnich, G. 1983, Solar Phys., 82, 443

Bashkirtsev, V., \& Mashnich, G. 1984, Solar Phys., 91, 93

Bommier, V., Leroy, J.-L., \& Sahal-Brechot, S. 1986, A\&A, 156, 79

Boyd, T. J. M., \& Sanderson, J. J. 1969, Plasma Dynamics (Nelson, London)

Cowling, T. G. 1976, Magnetohydrodynamics, Second Edn. (Adam Hilger, Bristol)

Cui Shu, Hu Ju, Ji Guo-Ping, et al. 1985, Chin. A\&A, 9, 49

Engvold, O. 1976, Solar Phys., 49, 238

Hirayama, T. 1986, in NASA CP-2442, Coronal and Prominence Plasmas, ed. A. I. Poland, 149

Hirayama, T., Nakagomi, Y., \& Okamoto, T. 1979, in Phys. Solar Prominences, ed. E. Jensen, P. Maltby, \& F. Q. Orrall, IAU Coll. 44, 48

Hirayama, T. 1971, Solar Phys., 17, 50

Hirayama, T. 1972, Solar Phys., 24, 310

Hirayama, T. 1985a, Solar Phys., 100, 415

Hirayama, T. 1985b, in Dyn. Quiescent Prominences, ed. V. Ruzdjak, \& E. Tandberg-Hanssen, IAU Coll., 117, 187

Jensen, E., \& Wiik, J. E. 1990, in Dyn. Quiescent Prominences, ed. V. Ruzdjak, \& E. Tandberg-Hanssen (Springer Verlag), 294

Kubot, J. 1980, in ed. A. F. Moriyama, \& J. C. Henoux, Proc. Japan - France Seminar on Solar Physics, 178 
Landman, D. A. 1984, ApJ, 279, 183

Landman, D. A. 1984, ApJ, 279, 438

Leroy, J.-L. 1985, in NASA Conf. Publ. 2374, Measurements of Solar Vector Magnetic Fields, ed. M. J. Hagyard, 121

Madjarska, M. S., Dermendjiev, V. N., Mouradian, Z., \& Nenovski, P. 1996, ApL. Comm., 34, 113

Malherbe, J. M., Schmieder, B., Ribes, E., \& Mein, P. 1983, A\&A, 119, 197

Mein, P., \& Mein, N. 1991, Solar Phys., 136, 317

Mein, P. 1977, Solar Phys., 54, 45

Murata, H. 1991, J. Plasma Phys., 46, 29

Nenovski, P. I., \& Dermendjiev, V. N. 1998, C. R. Acad. Sci. Bulgare, 51, 5

Nenovski, P., Dermendjiev, V. N., Madjarska, M. S., \& Vial, J.-C. 1998, in New Perspectives on Solar Prominences, ed. D. Webb, D. Rust, \& B. Schmieder, ASP Conf. Ser., 150, 354

Nenovski, P. 1996, Phys. Scr., 53, 345

Newton, R. S., Southwood, D. J., \& Hughes, W. J. 1978, Planet. Space Sci., 26, 201

Oliver, R. 1999, in ESA SP-448, Magnetic Fields and Solar Processes, ed. A. Wilson (Florence, Italy), 425

Priest, E. 1981, Solar Magnetohydrodynamics (D. Reidel Publishing Company, Dordrecht, Boston, London)

Schmieder, B., Malherbe, J. M., Mein, P., \& TandbergHanssen, E. 1984, A\&A, 136, 81

Schmieder, B., Malherbe, J. M., Poland, A. I., \& Simon, G. 1985, A\&A, 153, 64
Scholer, M. 1970, Planet. Space Sci., 18, 977

Shkarofsky, I. P., Johnson, T. W., \& Bachinski, M. P. 1996, The Particle Kinetics of Plasma (Addison Wesley, Reading, Massachusetts)

Tandberg-Hanssen, E. 1995, The Nature of Solar Prominences (Kluwer Academic Publishers)

Thompson, W., \& Schmieder, B. 1991, A\&A, 243, 501

Tsubaki, T., \& Takeuchi, A. 1986, Solar Phys., 104, 313

Tsubaki, T., Toyoda, M., Suematsu, Y., \& Gamboa, G. 1988, PASJ, 40, 121

Tsubaki, T. 1989, in Solar and Stellar Coronal Structures and Dynamics, National Solar Observatory, ed. R. C. Altrock, 140

Vernazza, J. E., Avertt, E. H., \& Loeser, R. 1981, ApJS, 45, 635

Vial, J.-C. 1982, ApJ, 253, 330

Vial, J.-C. 1986, in ed. A. I. Poland, NASA CP-2442, Coronal and Prominence Plasmas, 89

Vrsnak, B., \& Ruzdjak, V. 1994, in Solar Coronal Structures (VEDA Publ. House, Tatranska Lomnica), ed. V. Rusin, P. Heinzel, \& J.-C. Vial, IAU Coll., 144, 329

Vrsnak, B. 1993, Hvar Obs. Bull., 17, 23

Wiehr, E., Stellmacher, G., \& Balthasar, H. 1984, Solar Phys., 94,285

Yi, Z., Engvold, O., \& Keil, S. 1991, Solar Phys., 132, 63

Zhang, Q. Z., Livingston, W. C., Hu, J., \& Fang, C. 1987, Solar Phys., 114, 245

Zirin, H., \& Severny, A. B. 1961, Observatory, 81, 155 